\title{
Surgical Treatment of Compressive Radial Neuropathy Presenting with Motor Weakness and Pain: A Case Report
}

\author{
Gwangyoon Choi, Jinseo Yang, Yongjun Cho, Hyukjai Choi, Jinpyeong Jeon, Sukhyung Kang \\ Department of Neurosurgery, Chuncheon Sacred Heart Hospital, Hallym University College of Medicine, Chuncheon, \\ Republic of Korea
}

Corresponding author: Jinseo Yang Department of Neurosurgery, Chuncheon Sacred Heart Hospital, Hallym University College of Medicine, 77, Sakju-ro, Chuncheon 24253, Republic of Korea

Tel: +82-33-240-5171

Fax: +82-70-5096-8691

E-mail: pnsurgery@gmail.com

Received: May 18, 2020

Revised: June 8, 2020

Accepted: June 29, 2020
Compressive radial neuropathy, a type of nerve entrapment, can cause pain, extensor weakness, and sensory change. Usually, clinicians draw a distinction between posterior interoposterior interosseous nerve (PIN) syndrome in which weak extensor function is the main symptom, and radial tunnel syndrome (RTS), which causes pain but not motor weakness. Here, we present a case of a 55-year-old patient who experienced pain and tenderness in his right forearm, followed by extensor weakness, leading to finger and wrist drop. After undergoing surgical intervention, the patient showed improvement in both pain and motor weakness. This report indicates that clinical diagnosis should focus on clinical presentation before distinguishment as PIN syndrome or RTS. We suggest surgical intervention as an effective treatment for entrapment symptoms.

Key Words: Muscle weakness; Pain; Radial neuropathy

\section{INTRODUCTION}

Compressive radial neuropathy, a type of nerve entrapment, can cause pain, extensor weakness, and sensory change ${ }^{5)}$. The diagnosis and subclassification are largely based on clinical features, especially the patient's chief complaint. When pain in the forearm, specifically in the region innervated by radial nerve, is the patient's only symptom, clinicians give a diagnosis of radial tunnel syndrome (RTS). In contrast, when motor weakness from the weakened muscles governed by the radial nerve is the main symptom, posterior interosseous nerve (PIN) syndrome is considered a diagnosis. In the following case, the patient complains of both pain and motor weakness of forearm $^{6}$. Therefore, in the traditional way, the subject had both RTS and PIN syndrome. We present our experience of alleviation of both symptoms after surgery and suggest that the choice of treatment should be based on symptoms and mechanism as a diagnostic process, rather than the classification of syndromes. Additionally, for patients with radial neuropathy (RTS) whose symptoms, especially motor weakness, are refractory despite conservative treatment, i.e. steroid, we suggest timely surgical intervention as an effective treatment modality that leads to a better outcome. We present the following case in accordance with the CARE guidelines.

\section{CASE REPORT}

A 58-year-old male patient was referred to us by a local clinic. The patient complained of difficulty using his right hand properly and intractable pain in his right forearm. Pain in the posterior forearm started about 6 months ago. In addition, wrist and finger drop appeared suddenly 2 months later. He first tried treatment for herniated cervical disc disorder, which resulted in no improvement. He was a farmer with no underlying disease and had no history of trauma to his right arm.

\section{Physical Examination}

Tenderness of the posterior forearm, especially between the brachioradialis (BR) and the extensor carpi radialis brevis (ECRB) was noted. He showed right-sided motor weakness in wrist extension (grade 4), finger extension (grade 3), and supination (grade 4). This suggested radial nerve neuropathy. The patient also showed a radial shift during wrist extension, suggesting that extensor carpi radialis longus (ECRL) function was intact, and the affected lesion was located more distally from the elbow joint. Paresthesia was also present in his lateral proximal forearm, dorsum of the hand, and the $3^{\text {rd }}$ digit, largely corresponding to the region innervated by the radial nerve. 


\section{Diagnostic Imaging}

Magnetic resonance Imaging (MRI) showed abnormal enlargement and high T2 signal intensity in the patient's right radial nerve from his elbow joint extending to the PIN (Fig. 1). Muscle edema was also noted at the supinator, the extensor carpi ulnaris, the extensor digiti minimi, and the extensor digitorum communis. These findings were consistent with compressive radial neuropathy, affecting the nerve within the radial tunnel.

\section{Surgical Approach}

Under general anesthesia, we made a lazy-S incision between the $\mathrm{BR}$ and the ECRB. Retracting the BR showed compression of the superficial radial nerve (SRN), and surrounding adhesions were noted (Fig. 2A). After performing adhesiolysis, we explored proximally to expose the bifurcation of the SRN and the PIN, removing adhesions along the course. The PIN was severely compressed and pale-looking, so while following its course distally from the bifurcation, we tried to remove as much tissue as possible to get adequate decompression. The PIN appeared compressed at the arcade of Frohse as well (Fig. 2B), and after external neurolysis and decompression, we confirmed that the radial nerve and its branches were properly decompressed. The patient experienced no perioperative complications.

\section{Outcome}

Two weeks after surgery, his motor weakness improved from grade 4 to grade $4+$, and his pain disappeared. At the 1-month follow-up, his motor function of the forearm had returned to normal strength. Normal muscle tone in the innervated radial nerve was retained until his 3-month follow-up.

\section{DISCUSSION}

The radial nerve, extending from the posterior cord of brachial plexus, coursing through the spiral groove of the humerus to the forearm, passes a structure called the radial tunnel, then divides into 2 branches. This tunnel is bound by the BR, the ECRL and the ECRB laterally, and the tendon of the biceps brachii and the brachialis medially. The aforementioned nerve branches are: SRN, receiving sensory information from the posterior forearm, the dorsum of hand, and the dorsal aspect of the $1^{\text {st }}, 2^{\text {nd }}, 3^{\text {rd }}$, and lateral side of the 4 th digits; the PIN mainly providing extensor motor function to the forearm ${ }^{1)}$.

During its course, the radial nerve, especially the PIN, the deep motor branch of the radial nerve, can be compressed by one of the following structures: fibrous band tissue between the brachialis and the BR, recurrent radial vessels (or leash of Henry), the fibrous arch of the ECRB, proximal and distal

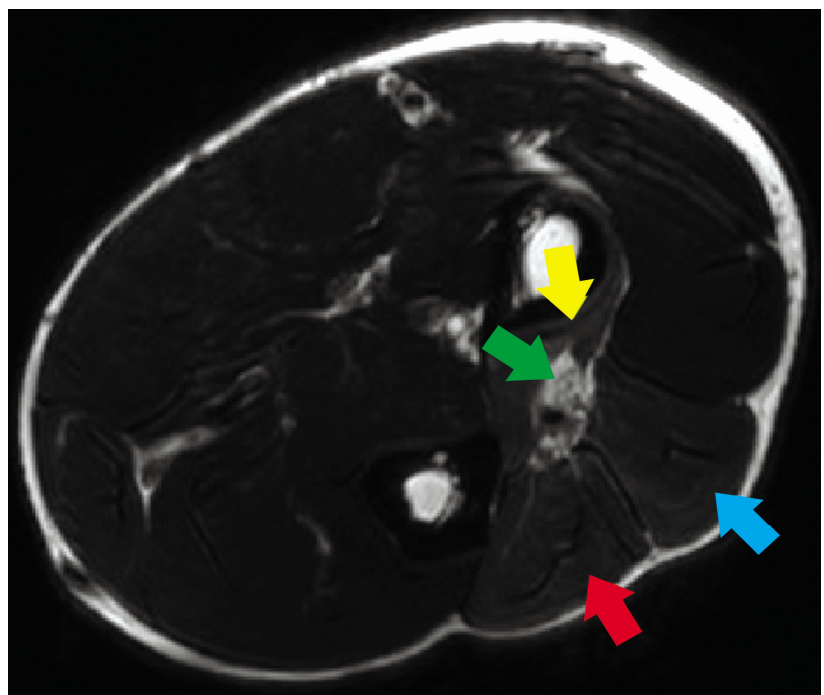

Fig. 1. Magnetic resonance image showed abnormal enlargement and high T2 signal intensity in the patient's right radial nerve from his elbow joint extending to the posterior interosseous nerve (annotated with green arrow). Muscle edema was also noted at the supinator (yellow arrow), the extensor carpi ulnaris (red arrow) and the extensor digitorum communis (blue arrow). These findings were consistent with compressive radial neuropathy, affecting the nerve within the radial tunnel.
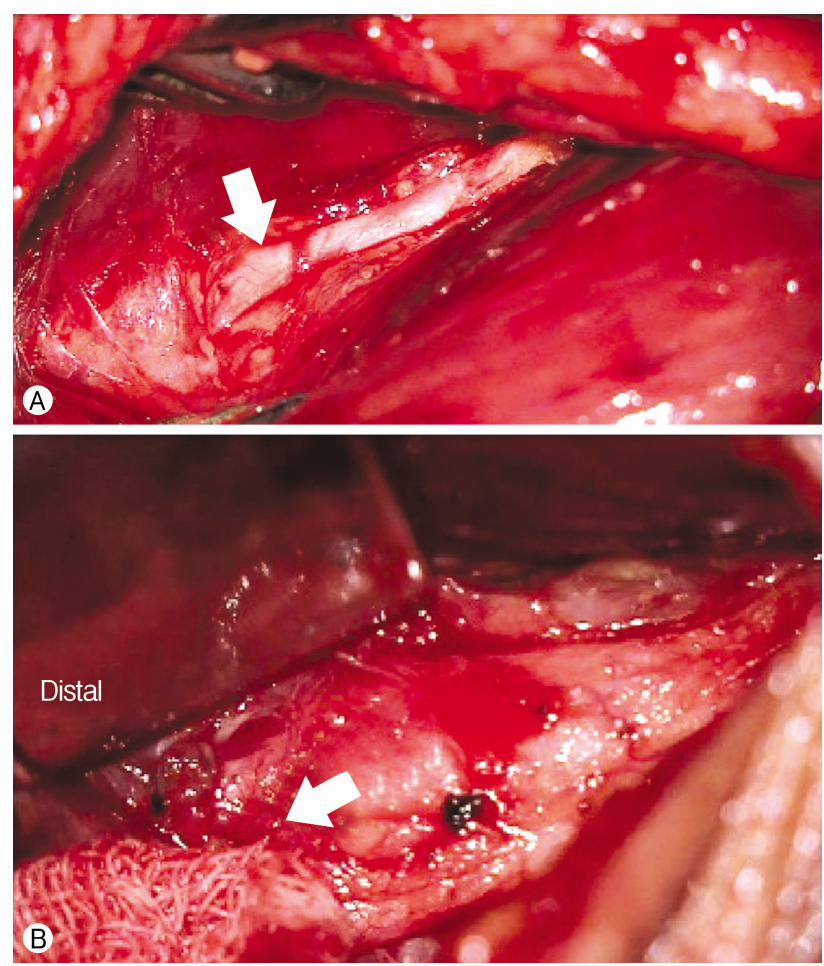

Fig. 2. Intraoperative findings are shown. The right side of the image corresponds to the proximal (elbow) side. (A) Compressed by nearby adhesion tissue, the proximal superficial radial nerve showed indentation (arrow), suggesting chronic injury. (B) By exploring further distally, the arcade of Frohse (arrow) appeared, pressing the posterior interosseous nerve, resulting in motor weakness. 
fibrous arches of the supinator. Among them, a fibrous arch at the proximal end of the supinator, also known as an arcade of Frohse, is the most common structure causing compressive neuropathy ${ }^{6}$.

As mentioned above, a distinction is usually drawn between PIN syndrome in which weak extensor function is the main symptom and RTS causing pain but no motor weaknesses ${ }^{3)}$. PIN syndrome is relatively considered an objectively evaluable disease, compared to RTS. Furthermore, RTS is considered a less well-known and controversial disease entity. This is partly due to the lack of electrodiagnostic proof of the existence and severity ${ }^{3)}$. Another possible reason for controversy is the inability to demonstrate the pathology. Unlike the suggestion of some that irritated PIN causes RTS ${ }^{3)}$, it is almost always impossible to demonstrate the corresponding irritant in the surgical field ${ }^{6}$.

In our case, decompressing the PIN and the SRN from adjacent adhesion tissues resulted in the relief of both pain and motor weakness. The symptom improvement of PIN syndrome from surgery is relatively explainable and is in alignment with various other studies. The theory for alleviation of RTS is 2fold. First, as pointed out in previous studies, freeing the PIN from adhesion tissues resulted in the absence of pain. Second, decompressing the SRN may have resulted in pain relief. The most well-known form of SRN compression, Wartenberg's syndrome, presents as a painful sensation in the region innervated by the SRN. The differences between RTS and Wartenberg's syndrome are the regions affected and the compressed location of the radial nerve. In Wartenberg's syndrome, SRN in the middle forearm is affected ${ }^{2}$, whereas in RTS, just as our patient's case, the radial nerve is compressed at the bifurcation of the radial nerve. Although the compressed location was different, maybe the patient experienced symptom relief from surgical decompression of the SRN just as a patient with Wartenberg's syndrome experiences improvement after surgery ${ }^{4)}$.

One thing to point out is that the choice of treatment should largely be based upon the patient's clinical presentation and the mechanism of symptom development. If our patient did not experience motor impairment symptoms, as surgery in RTS still lacks clinical evidence, he would not have had a chance to get total pain relief ${ }^{()}$. Localizing the lesion led us to perform the best possible treatment for the patient and resulted in clinical improvement. Subclassifying the patient's disease would not have been helpful in the process.

\section{CONCLUSION}

In the diagnosis of compressive radial neuropathy, neurologic examination is crucial, and MRI would be helpful for confirmation. If the patient's symptoms do not improve with conservative management, surgical decompression, and external neurolysis of the radial nerve may result in a favorable outcome.

\section{CONFLICTS OF INTEREST}

No potential conflict of interest relevant to this article was reported.

\section{REFERENCES}

1. Agarwal A, Chandra A, Jaipal U, Saini N: A panorama of radial nerve pathologies- an imaging diagnosis: A step ahead. Insights Imaging 9:1021-1034, 2018

2. Balakrishnan C, Bachusz RC, Balakrishnan A, Elliot D, Careaga D: Intraneural lipoma of the radial nerve presenting as Wartenberg syndrome: A case report and review of literature. Can J Plast Surg 17:e39-e41, 2009

3. Kim DH, Murovic JA, Kim YY, Kline DG: Surgical treatment and outcomes in 45 cases of posterior interosseous nerve entrapments and injuries. J Neurosurg 104:766-777, 2006

4. Lanzetta M, Foucher G: Entrapment of the superficial branch of the radial nerve (Wartenberg's syndrome). A report of 52 cases. Int Orthop 17:342-345, 1993

5. Mülkoğlu C, Nacır B, Genç H: Acute compressive radial neuropathy and wrist drop due to the repetitive overuse of the arm. Neurol Sci 41:989-991, 2020

6. Naam NH, Nemani S: Radial tunnel syndrome. Orthop Clin North Am 43:529-536, 2012 